\title{
Constrained Dynamic Controller Design for Ship Steering Yaw-Control
}

Wen-Jer Chang

Associate Professor, Department of Marine Engineering and Technology, National Taiwan Ocean Universiry. Keelung. Taiwan, R.O.C.

Follow this and additional works at: https://jmstt.ntou.edu.tw/journal

Part of the Engineering Commons

\section{Recommended Citation}

Chang, Wen-Jer (1997) "Constrained Dynamic Controller Design for Ship Steering Yaw-Control," Journal of Marine Science and Technology. Vol. 5: Iss. 1, Article 10.

DOI: 10.51400/2709-6998.2541

Available at: https://jmstt.ntou.edu.tw/journal/vol5/iss1/10

This Research Article is brought to you for free and open access by Journal of Marine Science and Technology. It has been accepted for inclusion in Journal of Marine Science and Technology by an authorized editor of Journal of Marine Science and Technology. 


\section{Constrained Dynamic Controller Design for Ship Steering Yaw-Control}

\section{Acknowledgements}

The authors wish to express sincere thanks to the anonymous referees for their constructive comments and suggestions which have made substantial improvements on this paper. This work was supported by the National Science Council of the Republic of China under Contract NSC86-2213-E-019-005. 


\title{
CONSTRAINED DYNAMIC CONTROLLER DESIGN FOR SHIP STEERING YAW-CONTROL
}

\author{
Wen-Jer Chang*
}

Keywords: Robust performance, Variance constraints, Pole assignment, Ship steering yaw-control.

\section{ABSTRACT}

Multiple constrained dynamic controller design problems associated with steering of ship yaw motion are discussed in this paper. The multiple constraints considered here contain individual state variance constraints, robust performance $\left(\boldsymbol{H}_{\infty}\right.$ norm) constate variance constraints, robust performance $\left(H_{\infty}\right.$ norints and regional pole assignment ( $D$-stability) constraints. The necessary and sufficient conditions for the existence of the constrained dynamic controller, which achieves the above three constraints, will be derived. Under achieving these conditions, the dynamic controllers will be solved by using the well known singular value decomposition (SVD) techniques and the theory of generalized inverses. Finally, a numerical simulation is given to show the usefulness and applicability of the present approach.

\section{INTRODUCTION}

In recent years, the problem of controlling surface ships in maneuvering situations has been receiving more and more attention from the operational safety and environmental viewpoints [1-10]. For ship steering, the stochastic disturbances are waves, wind gusts and obervation errors; and the deterministic disturbances are current, mean wind and curvature of the prespecified track. If the ship is modelled as a linear system, if the stochastic disturbances are supposed to be Gaussian noise, and if the risk to be minimized is the expection of the sum of a sequence of quadratic cost functions, then the solution to the ship yaw-control problem is known to be an adaptive controller [2-3] and a linear optimal controller [8-10]. However, the controllers of these literatures are derived to minimize a performance index, which consider the variance constraints only. Hence, in this paper we will deal with the multiple constrained

Paper Received June, 1996. Revised May 1997. Accepted May, 1997. Author for Correspondence: Wen-Jer Chang.

*Associate Professor, Department of Marine Engineering and Technology, National Taiwan Ocean University, Keelung, Taiwan, R.O.C. controller design problem for the ship yaw-control steering. Besides variance constraints, the robust performance and pole assignment constraints will be considered in this paper.

Regardless of what design technique is used, controllers are always designed based on information about the dynamic behavior of the process. This information (i.e., the "model") can have the form of a system of coupled partial differential equations or be simply the process gain and the settling time experienced by the plant operator. The accuracy of this information varies but is never perfect. Moreover, the behavior of the plant itself changes with time and these changes are rarely captured in the models. It is most desirable that the controller be insensitive to this kind of model uncertainty, i.e., the controller should be robust. A suitable norm that can incorporate both signal gain and robustness to plant uncertainty is the $\boldsymbol{H}_{\infty}$ norm. Therefore, the $\boldsymbol{H}_{\infty}$ control [11-16] for disturbance attenuation have a great deal of attention in the linear systems. Furthermore, the dynamics of linear systems is influenced by the location of its poles. A well-known desired region for dynamic systems is a disk $D$ centered at $(-\alpha, 0)$ with radius $\rho$, where $\alpha>0, \rho>0$ and $\alpha>\rho$ (Fig. 1). The pro-blem of locating all the closed-loop poles of controlled systems inside a specified disk $D(\alpha, \rho)$ is known as "D-stability problem" [17-20]. Considering the above performance requirements, the multiple constrained controller design problem in this paper will deal with variance constraints (which has been considered in [8-10]), robust performance constraints [11-16] (i.e., $\boldsymbol{H}_{\infty}$ norm constraints) and $D$-stability constraints [17-20] (i.e., pole assignment constraints) for ship steering yaw-control, simultaneously.

The approach of this paper is based on the upper bound covariance assignment technique, which has been developed by the authors in [16, 21-22] and [15, 23] for linear continuous-time and discrete-time stochastic systems, respectively. This technique provide the necessary and sufficient conditions as well as 
controller solutions for assigning the upper bound covariance matrix, whose diagonal elements satisfies the individual variance constraints for closed-loop systems. However, in [15-16, 21-23] the pole assignment constrained design problem has not been considered. Hence, the purpose of this paper is using the upper bound covariance assignment technique to derive a strategy to design an output feedback dynamic controller such that the closed-loop ship steering yaw-control systems achieve prespecified three constraints: individual state variance constraints, robust performance constraints and $D$-stability constraints.

\section{DYNAMIC MODEL OF SHIP YAW MOTION}

The development of the linearized, state-variable equations of motion for a ship moving in the horizontal plane presented here is obtained from Newton's laws expressing conservation of linear and angular momentum. It is customary to write the equations using a coordinate frame fixed to the ship (see Fig. 2). For a ship like a tanker there is little coupling between the different modes and the steering dynamics can therefore be described by considering the surge, sway and yaw motions separately. The equations of motion can be written as

$$
\begin{aligned}
& m\left(\dot{u}-\dot{v} r-x_{G} r^{2}\right)=X, \\
& m\left(\dot{v}+u r+x_{G} \dot{r}\right)=\Upsilon \\
& I_{Z} \dot{r}+m x_{G}(\dot{v}+r u)=N,
\end{aligned}
$$

where $\boldsymbol{m}$ is the mass of the ship with $I_{Z}$ being its moment of inertia with respect to the $z$-axis and $x_{G}$ being the $x$-coordinate of the centre of mass. The projections of the total ship speed on the $x$ - and $y$-axes are the surge velocity $u(t)$ and the sway velocity $v(t)$. The yaw rate is denoted by $r(t)=d \psi(t) / d t$, where $\psi(t)$

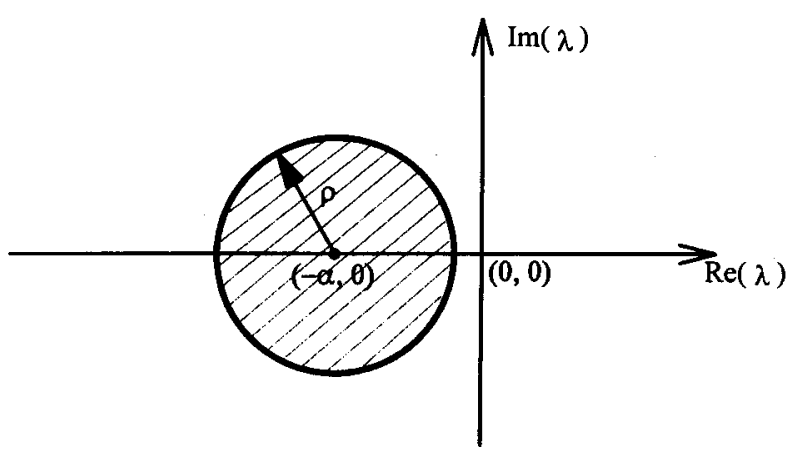

the is the heading angle. Moreover, the right-hand sides of Eqs. (1)-(3) are the hydrodynamic forces and moments, i.e., $X$ and $Y$ are the components of the hydrodynamic forces on the $\mathrm{x}$ - and $\mathrm{y}$-axes, $N$ is the zcomponent of the hydrodynamic moment.

The linearized dynamic equations of motion Eqs. (1)-(3) are easily converted to state-space form by solving for the derivatives $\boldsymbol{v}(t)$ and $\dot{r}(t)$. This gives the following dynamic model for the yaw motion of the ship:

$$
\begin{aligned}
{\left[\begin{array}{c}
\dot{r}(t) \\
\dot{\psi}(t)
\end{array}\right] } & =\left[\begin{array}{cc}
-1 / T_{s} & 0 \\
1 & 0
\end{array}\right]\left[\begin{array}{l}
\boldsymbol{r}(t) \\
\psi(t)
\end{array}\right] \\
& +\left[\begin{array}{c}
K_{s} / T_{s} \\
0
\end{array}\right] \delta(t)+\left[\begin{array}{l}
e_{1}(t) \\
e_{2}(t)
\end{array}\right], \\
& =\mathbf{A}\left[\begin{array}{l}
r(t) \\
\psi(t)
\end{array}\right]+\mathbf{B} \delta(t)+\mathbf{D}\left[\begin{array}{l}
e_{1}(t) \\
e_{2}(t)
\end{array}\right],
\end{aligned}
$$

where $\delta(t)$ is the rudder angles of the ship, and $T_{s}<0$ and $\boldsymbol{K}_{\boldsymbol{s}}>0$ are time constants for the ship yaw motion [9]. In Eq. (4), the roll motion is neglected without loss of generality [9]. The $e_{1}(t)$ and $e_{2}(t)$, which depend on the sea level and wave slope, denote the forces generated by the wind and wave. These forcing terms can be considered as the sum of constant and random components. Because of the long time constants of ships it is reasonable to approximate the random components by white noise both for wind- and wave-generated forces. It was shown in [9] that the forcing terms $e_{1}(t)$ and $e_{2}(t)$ may be generated as zero-mean white noises by a differential equation system (see (3.10) in [9]) for the numerical simulations. Note that the system Eq. (4) is unstable since the time constant $\boldsymbol{T}_{\boldsymbol{s}}$ is a negative scalar.

In [3], the LQG (Linear-Quadratic-Gaussian) control theory has been used to find an optimal regulator such that the following performance function:

$$
J=\frac{1}{T_{J}} \int_{0}^{T_{J}}\left[\psi^{2}(t)+q \delta^{2}(t)\right] d t,
$$

where $q$ is a weighting scalar, is minimized. From [3],

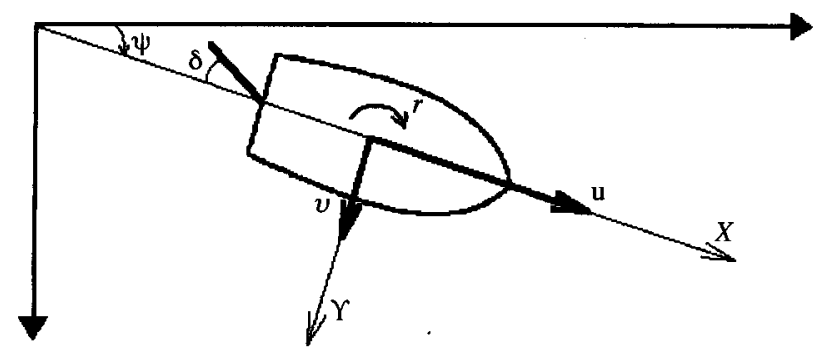


it can be find that the criterion Eq. (5) consists a state feedback from heading and yaw rate only, i.e., a PDregulator. The LQG controllers minimize a linear quadratic performance index Eq. (5), which lacks guaranteed variance constraints with respect to individual system states. Furthermore, the state variance constraint is the only one considered for the ship steering control problems in the previous literature. In the literature [1-10], there has been still little multiple constrained controller design approach for the ship steering. Hence, in the following section we will develop a methodology to find a dynamic controller which achieve multiple constraints for the ship yaw motion. These constraints considered in this paper are individual state variance constraints, closed-loop transfer function $\boldsymbol{H}_{\infty}$ norm constraints and regional closed-loop pole constraints.

\section{CONTROLLER DESIGN USING UPPER BOUND COVARIANCE ASSIGNMENT TECHNIQUE}

Consider the linearized steering model Eq. (4) of the ship yaw motion. It is assumed that the output signals of this system are $r(t)$ and $\psi(t)$. Then, the system output may be written as

$$
y(t)=\left[\begin{array}{l}
r(t) \\
\psi(t)
\end{array}\right] .
$$

In this paper, we use the following dynamic output feedback controller to drive the unstable system Eq. (4):

$$
\begin{aligned}
& \dot{x}_{c}(t)=\mathbf{A}_{c} x_{c}(t)+\mathbf{B}_{c} y(t), \\
& \delta(t)=\mathbf{C}_{c} x_{c}(t)+\mathbf{D}_{c} y(t),
\end{aligned}
$$

where $\boldsymbol{x}_{\boldsymbol{c}}(\boldsymbol{t}) \in \mathbf{R}^{n_{c}}$, and $\mathbf{A}_{\mathbf{c}}, \mathbf{B}_{\mathrm{c}}, \mathbf{C}_{\mathbf{c}}$ and $\mathbf{D}_{\mathrm{c}}$ are constant matrices with appropriate dimensions. In practice, a complete set of state variable directly available for state feedback purpose may not always exist. Consequently, it is worthy of using dynamic output feedback controllers with any order from a practical and engineering viewpoint.

Combining Eqs. (4) and (7), the closed-loop system for ship yaw motion has the following form:

$$
\begin{aligned}
& \dot{x}(t)=\hat{\mathbf{A}} x(t)+\tilde{\mathbf{D}}\left[\begin{array}{l}
e_{1}(t) \\
e_{2}(t)
\end{array}\right], \\
& y(t)=\widetilde{\mathbf{C}}_{y} x(t),
\end{aligned}
$$

where

$$
\boldsymbol{x}(\boldsymbol{t}) \equiv\left[\begin{array}{c}
\boldsymbol{r}(\boldsymbol{t}) \\
\boldsymbol{\psi}(\boldsymbol{t}) \\
\boldsymbol{x}_{c}(t)
\end{array}\right], \tilde{\mathbf{A}} \equiv\left[\begin{array}{ccc}
-1 / \boldsymbol{T}_{s} & 0 & 0 \\
1 & 0 & 0 \\
0 & 0 & 0
\end{array}\right],
$$

$$
\begin{gathered}
\widetilde{\mathbf{B}} \equiv\left[\begin{array}{cc}
\mathbf{K}_{\mathbf{s}} / T_{s} & 0 \\
0 & 0 \\
0 & \mathbf{I}_{n_{c}}
\end{array}\right], \mathbf{G} \equiv\left[\begin{array}{l}
\mathbf{D}_{\mathbf{c}} \mathbf{C}_{\mathbf{c}} \\
\mathbf{B}_{\mathbf{c}} \mathbf{A}_{\mathbf{c}}
\end{array}\right], \\
\widetilde{\mathbf{C}} \equiv\left[\begin{array}{lll}
1 & 0 & 0 \\
0 & 1 & 0 \\
0 & 0 & \mathbf{I}_{n_{c}}
\end{array}\right]=\mathbf{I}_{n_{c}+2}, \tilde{\mathbf{D}} \equiv\left[\begin{array}{ll}
1 & 0 \\
0 & 1 \\
0 & 0
\end{array}\right], \\
\tilde{\mathbf{C}}_{y} \equiv\left[\begin{array}{lll}
1 & 0 & 0 \\
0 & 1 & 0
\end{array}\right], \tilde{\mathbf{A}} \equiv \tilde{\mathbf{A}}+\tilde{\mathbf{B}} \mathbf{G} \tilde{\mathbf{C}},
\end{gathered}
$$

where $I_{n}$ denote the identity matrix with dimension $n \times n$. For the closed-loop system Eq. (8), the closedloop transfer function $\boldsymbol{H}(s)$ from noise input vector $\left[\begin{array}{lll}e_{1}(t) & e_{2}(t)\end{array}\right]^{T}$ to output $y(t)$ may be written as

$$
\boldsymbol{H}(s) \equiv \tilde{\mathbf{C}}_{\boldsymbol{y}}(s \mathrm{I}-\hat{\mathbf{A}})^{-1} \tilde{\mathbf{D}} .
$$

Moreover, let us define $\mathbf{X}$ as the steady state covariance matrix of state vector of the closed-loop system (8), i.e., $X \equiv \lim _{t \rightarrow \infty} E\left[x(t) x(t)^{T}\right]$, where $E[$. $]$ denotes the expection of [.]. It is well known that this state covariance matrix satisfies the following Lyapunov equation:

$$
\widehat{\mathbf{A}} \mathbf{X}+\mathbf{X} \widehat{\mathbf{A}}^{T}+\tilde{\mathbf{D}} \tilde{\mathbf{E}} \tilde{\mathbf{D}}^{T}=0,
$$

where $\widetilde{\mathbf{E}}$ denotes the covariance matrix of noise input vector $\left[e_{1}(t) e_{2}(t)\right]^{T}$.

\section{Definition 1}

The closed-loop system Eq. (8) is said to be $D(\alpha, \rho)$-stability if all the eigenvalues $\lambda_{i}$ of state dynamic matrix Â. satisfy

$$
\left|\lambda_{i}-\alpha\right|<\rho
$$

\section{Lemma 1}

Consider closed-loop ship steering system (8). Let $\mathbf{G}$ be given and let $\gamma>0$ be a fixed scalar. If there exists a positive definite matrix $\overline{\mathbf{X}}$ satisfying

$$
\begin{aligned}
& \hat{\mathbf{A}} \overline{\mathbf{X}}+\overline{\mathbf{X}} \hat{\mathbf{A}}^{T}+\gamma^{-2} \overline{\mathbf{X}} \tilde{\mathbf{R}} \overline{\mathbf{X}}+\tilde{\mathbf{D}} \tilde{\mathbf{E}} \tilde{\mathbf{D}}^{T}+\alpha^{-1} \hat{\mathbf{A}} \overline{\mathbf{X}} \hat{\mathbf{A}}^{T} \\
& \quad+\alpha^{-1}\left(\alpha^{2}-\rho^{2}\right) \overline{\mathbf{X}}=0
\end{aligned}
$$

where $\tilde{\mathbf{R}}=\tilde{\mathbf{C}}_{\boldsymbol{y}}^{T} \tilde{\mathbf{C}}_{\boldsymbol{y}}$. Then, the closed-loop system Eq. (8) is $D(\alpha, \rho)$-stable, the norm of closed-loop transfer function satisfies $\|\boldsymbol{H}(s)\|_{\infty} \leq \gamma$ and the state covariance matrix satisfies $\mathbf{X} \leq \overline{\mathbf{X}}$.

\section{Proof:}

It is assumed that $\lambda$ and $p$ are eigenvalue and right eigenvector of $\hat{\mathbf{A}}^{T}$, respectively. Letting and 
putting it into Eq. (13), we have

$$
\begin{aligned}
& {\left[\alpha^{-1}\left(\boldsymbol{x}^{2}+2 \boldsymbol{x} \alpha+\alpha^{2}+\boldsymbol{y}^{2}-\rho^{2}\right)\right] \boldsymbol{p}^{*} \overline{\mathbf{X}} \boldsymbol{p}} \\
& \quad=-\boldsymbol{p}^{*}\left(\boldsymbol{\gamma}^{-2} \overline{\mathbf{X}} \tilde{\mathbf{R}} \overline{\mathbf{X}}+\tilde{\mathbf{D}} \tilde{\mathbf{E}} \tilde{\mathbf{D}}^{T}\right) \boldsymbol{p} .
\end{aligned}
$$

Since $\overline{\mathbf{X}}>0$, and $\gamma^{2} \overline{\mathbf{X}} \tilde{\mathbf{R}} \overline{\mathbf{X}}+\widetilde{\mathbf{D}} \tilde{\mathbf{E}} \tilde{\mathbf{D}}^{T} \geq 0$, from Eq. (14) we obtain

$$
(x+\alpha)^{2}+y^{2} \leq \rho^{2},
$$

which means that all eigenvalues of $\hat{\mathbf{A}}$ should be located in a specified disk $D(\alpha, \rho)$.

Consider Eq. (13), from the Lemma 1 of [24] we may obtain $\|\boldsymbol{H}(s)\|_{\infty} \leq \gamma$ since $\tilde{\mathbf{D}} \tilde{\mathbf{E}} \tilde{\mathbf{D}}^{T}+\alpha^{-1} \hat{\mathbf{A}} \overline{\mathbf{X}} \hat{\mathbf{A}}^{T}$ $+\alpha^{-1}\left(\alpha^{2}-\rho^{2}\right) \overline{\mathbf{X}} \geq 0$. Subtracting Eq. (11) from Eq. (13) and using $\gamma^{-2} \overline{\mathbf{X}} \tilde{\mathbf{R}} \overline{\mathbf{X}}+\alpha^{-1} \hat{\mathbf{A}} \overline{\mathbf{X}} \hat{\mathbf{A}}^{T}+\alpha^{-1}\left(\alpha^{2}-\rho^{2}\right)$ $\overline{\mathbf{X}} \geq 0$, the inequality $\mathbf{X} \leq \overline{\mathbf{X}}$ will be got from the proofs of Theorem 4.2 of [17] due to the fact that $\hat{\mathbf{A}}$ is stable.

From Lemma 1, we may conclude that the constrained dynamic controller design problem for ship steering yaw motion is to determine the dynamic controller such that the following performance criteria are satisfied.

\section{$<1>$ Individual variance constraints:}

$$
[\mathbf{X}]_{i i} \leq \sigma_{i}^{2}, i=1,2,
$$

where []$_{i i}$ denotes the th diagonal element of matrix [•] and $\sigma_{i}^{2}$ denote the Root-Mean-Squared (RMS) constraints for the individual variances of the linearized ship steering system states, i.e., states $r(t)$ and $\psi(t)$ in Eq. (4).

$<2>H_{\infty}$ norm constraints:

$$
\|\boldsymbol{H}(s)\|_{\infty} \leq \gamma,
$$

for some prescribed positive constant $\gamma$.

$<3>D(\alpha, \rho)$-stability constraints:

$$
\lambda(\overline{\mathbf{A}}) \in D(\alpha, \rho),
$$

where $\lambda(\bullet)$ denotes any eigenvalues of matrix $[\bullet]$.

\section{Definition 2}

Given a desired circular region $D(\alpha, \rho)$ and a positive constant $\gamma$. Let $\overline{\mathbf{X}}=\overline{\mathbf{X}}^{T}>0$ be a positive definite prespecified matrix which meets performance constraint $<1\rangle$. Then $\overline{\mathbf{X}}$ is called a $D-\gamma$-assignable matrix if there exists a set of matrixes $\mathbf{G}$ such that Eq. (13) has the positive definite solution $\overline{\mathbf{X}}$.

\section{Theorem 1}

A specified positive definite upper bound covariance matrix $\overline{\mathbf{X}}=\overline{\mathbf{X}}^{T}>0$ satisfying performance constraint $<1>$ (i.e., $[\overline{\mathbf{X}}]_{i i} \leq \sigma_{i}^{2}, i=1,2$ ) is $D-\gamma$-assignable by some $\mathbf{G}$ if and only if

$$
\begin{aligned}
& \left(\alpha^{-\frac{1}{2}} \tilde{\mathbf{A}}+\alpha^{\frac{1}{2}} \mathbf{I}\right) \overline{\mathbf{X}} \tilde{\mathbf{C}}^{T}\left(\tilde{\mathbf{C}} \overline{\mathbf{X}} \tilde{\mathbf{C}}^{T}\right)^{-1} \\
& \tilde{\mathbf{C}} \overline{\mathbf{X}}\left(\alpha^{-\frac{1}{2}} \tilde{\mathbf{A}}^{\boldsymbol{T}}+\alpha^{\frac{1}{2}} \mathbf{I}\right)-\overline{\mathbf{X}}_{\alpha \rho} \geq 0, \\
& \hat{\mathbf{B}} \overline{\mathbf{X}}_{\alpha \rho} \hat{\mathbf{B}}=0
\end{aligned}
$$

where $[\cdot]^{+}$denotes the Moore-Penrose inverse of matrix (see [25]) and

$$
\begin{aligned}
\hat{\mathbf{B}} & \equiv \mathbf{I}-\widetilde{\mathbf{B}} \tilde{\mathbf{B}}^{+} \\
& \begin{aligned}
\overline{\mathbf{X}}_{\alpha \rho} & \equiv \tilde{\mathbf{A}} \overline{\mathbf{X}}+\overline{\mathbf{X}} \tilde{\mathbf{A}}^{T}+\gamma^{-2} \overline{\mathbf{X}} \tilde{\mathbf{R}} \overline{\mathbf{X}}+\tilde{\mathbf{D}} \tilde{\mathbf{E}} \tilde{\mathbf{D}}^{T}+\alpha^{-1} \tilde{\mathbf{A}} \overline{\mathbf{X}} \tilde{\mathbf{A}}^{T} \\
& +\alpha^{-1}\left(\alpha^{2}-\rho^{2}\right) \overline{\mathbf{X}}
\end{aligned}
\end{aligned}
$$

\section{Proof:}

It is clear that Eq. (13) may be written as

$$
\begin{aligned}
& \left.\left[\alpha^{-\frac{1}{2}} \tilde{\mathbf{B}} \mathbf{G P}+\left(\alpha^{-\frac{1}{2}} \tilde{\mathbf{A}}+\alpha^{\frac{1}{2} \mathbf{I}}\right) \overline{\mathbf{X}} \tilde{\mathbf{C}}^{T}(\mathbf{P P})^{T}\right)^{-1} \mathbf{P}\right] \\
& {\left[\alpha^{-\frac{1}{2}} \tilde{\mathbf{B}} \mathbf{G P}+\left(\alpha^{-\frac{1}{2}} \tilde{\mathbf{A}}+\alpha^{\frac{1}{2}} \mathbf{I}\right) \overline{\mathbf{X}} \tilde{\mathbf{C}}^{T}\left(\mathbf{P} \mathbf{P}^{T}\right)^{-1} \mathbf{P}\right]^{T}} \\
& =\mathbf{K} \mathbf{K}^{\boldsymbol{T}},
\end{aligned}
$$

where $\mathbf{P} \mathbf{P}^{T}=\tilde{\mathbf{C}} \overline{\mathbf{X}} \tilde{\mathbf{C}}^{\boldsymbol{T}}$ and $\mathbf{K} \in \mathbf{R}^{\left(n_{c}+2\right) \times\left(n_{c}+2\right)}$ is defined by

$$
\begin{aligned}
& \left(\alpha^{-\frac{1}{2}} \tilde{\mathbf{A}}+\alpha^{\frac{1}{2}} \mathbf{I}\right) \overline{\mathbf{X}} \tilde{\mathbf{C}}^{\boldsymbol{T}}\left(\tilde{\mathbf{C}} \overline{\mathbf{X}} \tilde{\mathbf{C}}^{T}\right)^{-1} \\
& \tilde{\mathbf{C}} \overline{\mathbf{X}}\left(\alpha^{-\frac{1}{2}} \tilde{\mathbf{A}}^{\boldsymbol{T}}+\alpha^{\frac{1}{2}} \mathbf{I}\right)-\overline{\mathbf{X}}_{\alpha \rho}=\mathbf{K K} \mathbf{K}^{\boldsymbol{T}} .
\end{aligned}
$$

From Eq. (23), it can be found that the right-hand side of Eq. (24) is positive semidefinite, hence the condition Eq. (19) will be obtained, immediately. From Lemma 2.1 of [26], Eq. (23) is equivalent to

$$
\alpha^{-\frac{1}{2}} \widetilde{\mathbf{B}} \mathbf{G}=\mathbf{K V P} \mathbf{P}^{-1}-\left(\alpha^{-\frac{1}{2}} \tilde{\mathbf{A}}+\alpha^{\frac{1}{2} \mathbf{I}}\right) \overline{\mathbf{X}} \tilde{\mathbf{C}}^{T}\left(\mathbf{P P}^{T}\right)^{-1},
$$

where $\mathbf{V}$ is some orthogonal matrix. By assumption $\mathbf{G}$ satisfying Eq. (25) exists. From the theory of generalized inverses [27], Eq. (25) has to be solvable for $\mathbf{G}$, which is guaranteed if and only if

$$
\left(\mathbf{I}-\tilde{\mathbf{B}} \tilde{\mathbf{B}}^{+}\right)\left[\mathbf{K V P} \mathbf{P}^{-1}-\left(\alpha^{-\frac{1}{2}} \tilde{\mathbf{A}}+\alpha^{\frac{1}{2} \mathbf{I}}\right) \overline{\mathbf{X}} \tilde{\mathbf{C}}^{T}\left(\mathbf{P P}^{T}\right)^{-1}\right]=0,
$$

or $\left.\mathbf{I}-\widetilde{\mathbf{B}} \tilde{\mathbf{B}}^{+}\right) \mathbf{K V}=\left(\mathbf{I}-\tilde{\mathbf{B}} \tilde{\mathbf{B}}^{+}\right)\left(\alpha^{-\frac{1}{2}} \tilde{\mathbf{A}}+\alpha^{\frac{1}{2} \mathbf{I}}\right) \overline{\mathbf{X}} \tilde{\mathbf{C}}^{\boldsymbol{T}}\left(\mathbf{P} \mathbf{P}^{\boldsymbol{T}}\right)^{-1} \mathbf{P}$. 
Since $\mathbf{P}$ is nonsingular, using Lemma 2.1 of [26] and Eq. (21), Eq. (27) is equivalent to

$$
\begin{aligned}
& \hat{\mathbf{B}}\left[\mathbf{K} \mathbf{K}^{T}-\left(\alpha^{-\frac{1}{2}} \tilde{\mathbf{A}}+\alpha^{\frac{1}{2} \mathbf{I}}\right) \overline{\mathbf{X}} \tilde{\mathbf{C}}^{\boldsymbol{T}}\right. \\
& \left.\left(\tilde{\mathbf{C}} \overline{\mathbf{X}} \tilde{\mathbf{C}}^{\boldsymbol{T}}\right)^{-1} \tilde{\mathbf{C}} \overline{\mathbf{X}}\left(\alpha^{-\frac{1}{2}} \tilde{\mathbf{A}}+\alpha^{\frac{1}{2}} \mathbf{I}\right)\right] \hat{\mathbf{B}}=0 .
\end{aligned}
$$

From Eq. (24), we can find that Eq. (28) is equivalent to Eq. (20).

Using Theorem 1 and Definition 2 we know that if the necessary and sufficient conditions of Theorem 1 hold for a specified upper bound covariance matrix $\overline{\mathbf{X}}$, then there exist some matrices $\mathbf{G}$ such that Eq. (13) is satisfied. When Eq. (13) is satisfied, the performance constraints $\langle 1\rangle-\langle 3\rangle$ will meet from Lemma 1. That is, Theorem 1 provides the necessary and sufficient conditions for the existence of dynamic controllers $\mathbf{G}$ which achieve a suitable upper bound covariance matrix $\overline{\mathbf{X}}$ such that the closed-loop ship steering system Eq. (8) satisfies the multiple performance requirements $\langle 1\rangle-<3\rangle$. In the following theorem, the dynamic controller solutions $\mathbf{G}$ will be derived when the conditions of Theorem 1 are satisfied.

\section{Theorem 2}

If the conditions of Theorem 1 hold for a specified upper bound covariance matrix , then the dynamic controller which achieve this specified is given by

$$
\begin{aligned}
& \mathbf{G}=\tilde{\mathbf{B}}^{+}+\left[\alpha^{-\frac{1}{2}} \mathbf{K} \mathbf{V}_{\mathbf{k}}\left[\begin{array}{cc}
\mathbf{I} & 0 \\
0 & \mathbf{U}_{\mathrm{a}}
\end{array}\right] \mathbf{V}_{\boldsymbol{p}}^{\boldsymbol{T}} \mathbf{P}^{-1}\right. \\
& \left.-(\tilde{\mathbf{A}}+\boldsymbol{\alpha} \mathbf{I}) \overline{\mathbf{X}} \tilde{\mathbf{C}}^{\boldsymbol{T}}\left(\mathbf{P} \mathbf{P}^{\boldsymbol{T}}\right)^{-1}\right]+\left(\mathbf{I}-\tilde{\mathbf{B}}^{+} \tilde{\mathbf{B}}\right) \tilde{\mathbf{Z}},
\end{aligned}
$$

where $\mathbf{K}$ is expressed as in Eq. (23), $\tilde{\mathbf{Z}} \in \mathbb{R}^{\left(n_{c^{+}}+1\right) \times\left(n_{c^{+}}\right)}$ is an arbitrary matrix, $\mathbf{U}_{\mathbf{a}} \in \mathbf{R}^{\left(n_{c}+2-r_{k}\right) \times\left(n_{c}+2-r_{k}\right)}$ is an arbitrary orthogonal matrix, $\boldsymbol{r}_{\boldsymbol{k}}=\operatorname{Rank}(\widetilde{\mathbf{B} K})$, and $\mathbf{V}_{\mathbf{k}}$ and $\mathbf{V}_{\mathbf{p}}$ come from the SVD as follows:

$$
\begin{aligned}
& \tilde{\mathbf{B}} \mathbf{K}=\hat{\mathbf{U}} \Sigma \mathbf{V}_{\mathbf{k}}^{\boldsymbol{T}}, \\
& \hat{\mathbf{B}}\left(\alpha^{-\frac{1}{2}} \tilde{\mathbf{A}}+\alpha^{\frac{1}{2} \mathbf{I}}\right) \overline{\mathbf{X}} \tilde{\mathbf{C}}^{\boldsymbol{T}}\left(\mathbf{P P}^{\boldsymbol{T}}\right)^{-1} \mathbf{P}=\hat{\mathbf{U}} \Sigma \mathbf{V}_{\mathbf{p}}^{\boldsymbol{T}}
\end{aligned}
$$

\section{Proof:}

From the proofs of Theorem 1, it is known that the given $\overline{\mathbf{X}}$ is $D-\gamma$-assignable if and only if there exists a solution $\boldsymbol{G}$ to Eq. (25) for some orthogonal matrix $V$ satisfying Eq. (27). Using the theory of generalized inverses [27], the solutions $\boldsymbol{G}$ of Eq. (25) will be obtained by

$$
\mathbf{G}=\tilde{\mathbf{B}}^{+}\left[\alpha^{-\frac{1}{2}} \mathbf{K V P}-1-(\tilde{\mathbf{A}}+\alpha \mathbf{I}) \overline{\mathbf{X}} \tilde{\mathbf{C}}^{T}\left(\mathbf{P P}^{T}\right)^{-1}\right]
$$

$$
+\left(\mathbf{I}-\tilde{\mathbf{B}}^{+} \tilde{\mathbf{B}}\right) \tilde{\mathbf{Z}},
$$

where $\widetilde{\mathbf{Z}} \in \mathbb{R}^{\left(n_{c}+1\right) \times\left(n_{c}+2\right)}$ is an arbitrary matrix and $V$ is any orthogonal matrix satisfying Eq. (27). From Lemma 2.1 of [26], the general solutions $\boldsymbol{V}$ for Eq. (27) can be expressed as

$$
\mathbf{V}=\mathbf{V}_{\mathbf{k}}\left[\begin{array}{cc}
\mathbf{I} & 0 \\
0 & \mathbf{U}_{\mathrm{a}}
\end{array}\right] \mathbf{V}_{\boldsymbol{p}}^{\mathbf{T}},
$$

where $U_{a}$ is an arbitrary orthogonal matrix and $V_{k}$ and $\mathbf{V}_{\mathbf{p}}$ are defined in Eq. (30) and (31), respectively.

From the above theorems we know that the first step of designing the multiple constrained dynamic controllers is to assign a suitable upper bound covariance matrix $\overline{\mathbf{X}}$, which satisfies the performance constraints $<1>$, such that the conditions Eqs. (19) and (20) of Theorem 1 are satisfied. If the necessary and sufficient conditions of Theorem 1 are satisfied, then the dynamic controller solution $\mathbf{G}$ will be obtained from Eq. (29) of Theorem 2. In the following section, we will use the above upper bound covariance assignment technique to design the constrained dynamic controllers for the ship steering yaw motion. A numerical simulation will be also given in the following section.

\section{CONSTRAINED DYNAMIC CONTROLLER DESIGN AND SIMULATION FOR SHIP STEERING YAW MOTION}

In this section we will introduce the procedure of the constrained dynamic controller designing of ship steering yaw motion. Consider the linearized dynamic model of ship yaw motion Eq. (4), it is assumed that the forcing terms $e_{1}(t)$ and $e_{2}(t)$ are zero-mean white noise processes of intensities $\xi$ and $\mu$ respectively, i.e.,

$$
E\left[e_{1}(t)\right]=0, E\left[e_{1}(t) e_{1}(\tau)^{T}\right]=\xi \delta(t-\tau),
$$

and

$$
E\left[e_{2}(t)\right]=0, E\left[e_{2}(t) e_{2}(\tau)^{T}\right]=\xi \delta(t-\tau) .
$$

Then, the covariance matrix $\tilde{\mathbf{E}}$ of noise input vector $\left[\begin{array}{ll}e_{1}(t) & e_{2}(t)\end{array}\right]^{T}$ is

$$
\widetilde{\mathbf{E}}=\left[\begin{array}{cc}
\xi^{2} & \xi \mu \\
\xi \mu & \mu^{2}
\end{array}\right] \text {. }
$$

From Section III, we know that the first step of designing the constrained dynamic controllers, which achieve the multiple performance constraints $<1>-<3>$, is to assign a suitable upper bound covariance matrix $\overline{\mathbf{X}}$ such that constraint $<1>$ and conditions 
Eqs. (19)-(20) are satisfied. Without loss of generality, in this paper we consider that the ship steering model Eq. (4) is driven by the first order dynamic output controllers, i.e., $\boldsymbol{n}_{c}=1$ in Eq. (7). Then, the symmetric upper bound covariance matrix $\overline{\mathbf{X}}$ may be expressed as

$$
\overline{\mathbf{X}}=\left[\begin{array}{lll}
\bar{x}_{11} & \bar{x}_{12} & \bar{x}_{13} \\
\bar{x}_{12} & \bar{x}_{22} & \bar{x}_{23} \\
\bar{x}_{13} & \bar{x}_{23} & \bar{x}_{33}
\end{array}\right] .
$$

Consider the closed-loop ship steering model Eqs. (8)-(9) and Eq. (21), it is easy to obtain that for arbitrary $\boldsymbol{K}_{s}$ and $\boldsymbol{T}_{s}$, the elements of $\hat{\mathbf{B}}$ are all zero except the $(2,2)$ element. The value of the only one non-zero element of $\hat{\mathbf{B}}$ is always equivalent to 1 for arbitrary $\boldsymbol{K}_{s}$ and $\boldsymbol{T}_{\boldsymbol{s}}$ of $\tilde{\mathbf{B}}$. Putting $\hat{\mathbf{B}}$ matrix into condition Eq. $(20)$, we can conclude that the $(2,2)$ element of matrix $\overline{\mathbf{X}}_{\alpha \rho}$ in Eq. (20) must be zero and the remnant elements of $\overline{\mathbf{X}}_{\alpha \rho}$ may be arbitrary for satisfying the condition Eq. (20). Hence, the condition Eq. (20) will become

$$
\begin{aligned}
\gamma^{2} \bar{x}_{12}^{2} & +2 \bar{x}_{12}+\alpha^{-1} \bar{x}_{11}+\alpha^{-1}\left(\alpha^{2}-\rho^{2}\right) \bar{x}_{22} \\
& +\gamma^{-2} \bar{x}_{22}^{2}+\mu^{2}=0
\end{aligned}
$$

where the left-hand side of the Eq. $(38)$ is the $(2,2)$ element of matrix $\overline{\mathbf{X}}_{\alpha \rho}$. If we assign $\overline{\boldsymbol{x}}_{11}$ and $\overline{\boldsymbol{x}}_{22}$ to satisfy performance requirement $<1>$, i.e., $\bar{x}_{11} \leq \sigma_{1}^{2}$ and $\bar{x}_{22} \leq \sigma_{2}^{2}$, then the Eq. (38) will be a second order equation for the only one unknown variable $\overline{\boldsymbol{x}}_{12}$. Solving this second order equation, we may find a suitable variable $\bar{x}_{12}$ to satisfy condition Eq. (20) for the assigned values $\overline{\boldsymbol{x}}_{11}$ and $\overline{\boldsymbol{x}}_{22}$. Moreover, if we choose appropriate $\bar{x}_{33}, \bar{x}_{13}$ and $\bar{x}_{23}$ of $\overline{\mathbf{X}}$, it is easy to find a suitable positive definite matrix $\overline{\mathbf{X}}$ such that conditions Eqs. (19)-(20) are satisfied. The following simple procedure will provide the steps to design the multiple constrained dynamic controllers for the ship steering yaw-control problems.

Step 1: For given constraints $<1>-<3>$ (i.e., $\sigma_{1}, \sigma_{2}, \gamma$, $\alpha$ and $\rho$ are given), let us assign $\overline{\boldsymbol{x}}_{11}$ and $\overline{\boldsymbol{x}}_{22}$ to satisfy performance requirement $<1>$, i.e., $\overline{\boldsymbol{x}}_{11} \leq \sigma_{1}^{2}$ and $\overline{\boldsymbol{x}}_{22} \leq \sigma_{2}^{2}$.

Step 2: Solving the second order Eq. (38) to obtain $\bar{x}_{12}$.

Step 3: If the inequality $\bar{x}_{11} \bar{x}_{22}-\bar{x}_{12}^{2}>0$ is not satisfied, then slightly increase either of $\overline{\boldsymbol{x}}_{11}$ and $\overline{\boldsymbol{x}}_{22}$ and go to Step 2; otherwise, continue. Since this inequality is the necessary condition of $\overline{\mathbf{X}}>0$.

Step 4: Choosing appropriate $\bar{x}_{33}, \bar{x}_{13}$ and $\bar{x}_{23}$ such that $\overline{\mathbf{X}}>0$ and condition Eq. (19) is satisfied.
Step 5: Substituting $\overline{\mathbf{X}}$ into Eq. (24) to solve $\mathbf{K}$.

Step 6: Using SVD technique to find $V_{k}$ and $V_{p}$ from Eqs. (30) and (31), respectively.

Step 7: At last the dynamic controller $\mathbf{G}$ may be obtained from Eq. (29).

In this paper, a single screw/single rudder ro-ro ship was selected for the simulation study. The bow and stern draught is $11 \mathrm{~m}$ and the displacement is $52010 \mathrm{~m}^{3}$. The metacentric height is $0.45 \mathrm{~m}$. A diesel engine of $13000 \mathrm{HP}$ delivers the propulsion power. The ship is fitted with a four-bland propeller with a diameter of $6.3 \mathrm{~m}^{2}$. The detailed hull data, propeller data and rudder data of this test ship are given in the Table 1 of [9].

Consider the linearized dynamic equation of ship steering yaw motion Eq. (4), the time constants may be obtained from [9], i.e., $\boldsymbol{T}_{s}=-64 \mathrm{~s}$ and $\boldsymbol{K}_{\boldsymbol{s}}=0.04$ 1/s. Furthermore, form (3.10) of [9] we may obtain the forcing input noise terms $e_{1}(t)$ and $e_{2}(t)$, which have intensities $\xi=0.25$ and $\mu=-0.1$, respectively.

Without loss of generality, it is assumed that the system Eq. (4) is driven by a first order dynamic output controller. Then, from Eq. (9) we have

$$
\begin{aligned}
\mathbf{A} & =\left[\begin{array}{ccc}
0.01525 & 0 \\
1 & 0
\end{array}\right], \mathbf{B}=\left[\begin{array}{c}
-0.00625 \\
0
\end{array}\right], \\
\tilde{\mathbf{A}} & \equiv\left[\begin{array}{ccc}
0.015625 & 0 & 0 \\
1 & 0 & 0 \\
0 & 0 & 0
\end{array}\right], \\
\widetilde{\mathbf{B}} & =\left[\begin{array}{ccc}
-0.000625 & 0 \\
0 & 0 \\
0 & 1
\end{array}\right] .
\end{aligned}
$$

In this simulation, the multiple performance requirements $<1>-<3>$ of this paper will be assumed as follows:

$<1>$ Individual variance constraints: $\sigma_{1}^{2}=0.2$ and $\sigma_{2}^{2}=$ 0.1 , $<2>\boldsymbol{H}_{\infty}$ norm constraints: $\gamma=0.5$, $<3>D(\alpha, \rho)$-stability constraints: $\alpha=20$ and $\rho=18$.

From the above variance constraint $\langle 1\rangle$, we may assign the diagonal elements of upper bound covariance matrix $\overline{\mathbf{X}}$ to satisfy this constraint, i.e. we assign $\bar{x}_{11}=0.15<0.2$ and $\bar{x}_{22}=0.02<0.1$. Substituting $\overline{\boldsymbol{x}}_{11}$ and $\overline{\boldsymbol{x}}_{22}$ into Eq. (38) we may obtain $\overline{\boldsymbol{x}}_{12}=$ -0.0532133 . For satisfying condition (19) and the positive definite property of upper bound covariance matrix $\overline{\mathbf{X}}$, we choose $\bar{x}_{13}=0.02, \bar{x}_{23}=-0.01$ and $\bar{x}_{33}=0.5$. Then, the necessary and sufficient conditions Eqs. (19)-(20) of Theorem 1 are satisfied. Putting $\overline{\mathbf{X}}$ into Eq. (24) and Eqs. (30)-(31), we can calculate matrices $K, V_{k}$ and $V_{p}$, which will be used to solve the controller solutions G. Using Eq. (29) of Theorem 2, the controller gain matrix $\mathbf{G}$ will be calculated as 
$\mathbf{G}=\left[\begin{array}{ccc}7.553838 \times 10^{3} & 9.384469 \times 10^{3} & -0.875844 \times 10^{-2} \\ -0.618455 & -1.341565 & -2.00458\end{array}\right]$

That is, the dynamic output controller Eq. (7) has the following form:

$$
\dot{x}_{c}(t)=-2.00458 x_{c}(t)+[-0.618455-1.341565] y(t),
$$

and

$$
\begin{aligned}
& \delta(t)=-0.875844 \times 10^{-2} x_{c}(t)+\left[7.553838 \times 10^{3}\right. \\
& \left.9.384469 \times 10^{3}\right] y(t) .
\end{aligned}
$$

Figure 5 and Fig. 6 respectively present the responses of yaw rate and heading angle, which were driven by the zero-mean white noise inputs $e_{1}(t)$ and $e_{2}(t)$ (see Figs. 3 and 4). From the simulated responses Figs. 5 and 6 , we obtain the variances of system states as $E\left[r^{2}(t)\right]=7.97528 \times 10^{-3}$ and $E\left[\psi^{2}(t)\right]=$ $8.637664 \times 10^{-3}$. It can be found that the performance constraint $\langle 1\rangle$ is satisfied. Substituting the dynamic controller Eq. (40) and system parameters Eq. (39) into Lemma 4 of [12], it is obvious that the $\boldsymbol{H}_{\infty}$ norm of closed-loop transfer function satisfy the performance requirement $<2>$. Calculating the eigenvalues of the closed-loop dynamic state matrix $\widehat{\mathbf{A}}$, we find that the closed-loop system poles are -2.0046 , $-2.3528+j 0.5743$ and $-2.3528-j 0.5743$. These poles achieve the performance constraint $<3>$. From the above descriptions, it can be found that the dynamic output controller Eq. (41) drive the linearized ship steering system Eq. (4) to satisfy the multiple performance requirements $\langle 1\rangle-\langle 3\rangle$, simultaneously.

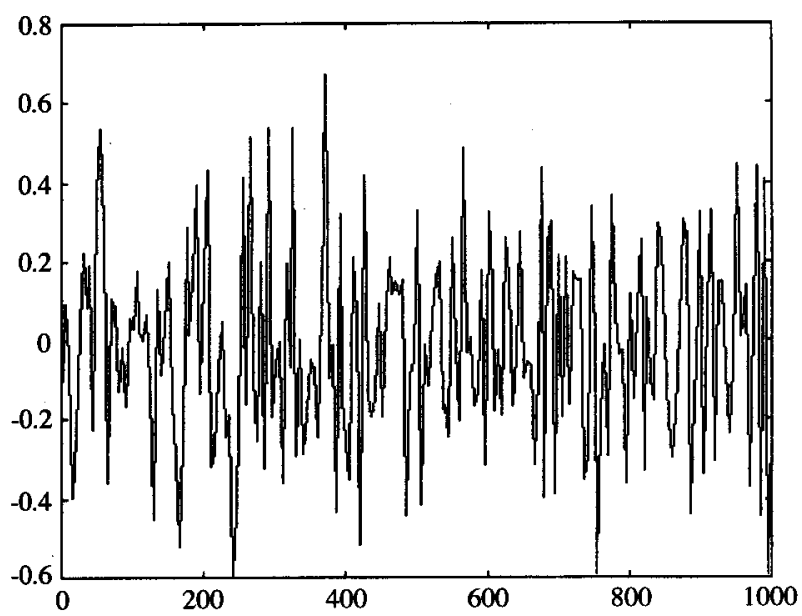

Fig. 3. White noise force input $e_{1}(t)$.

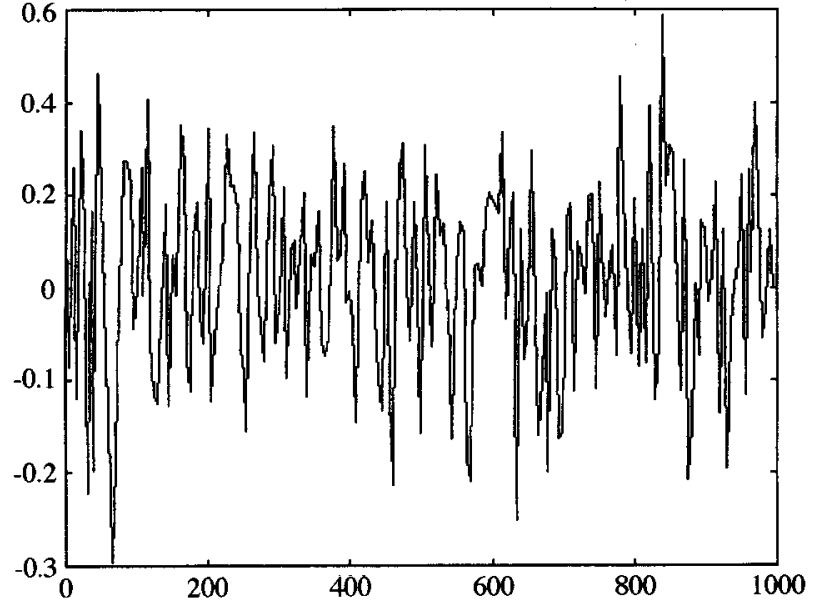

Fig. 4. White noise force input $e_{2}(t)$.

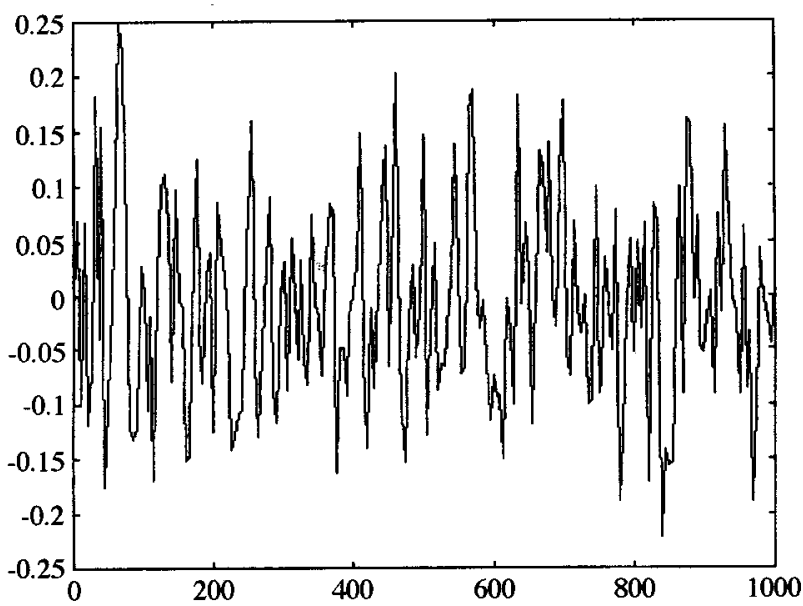

Fig. 5. Output response of yaw rate $r(t)$ controlled by dynamic controller (41).

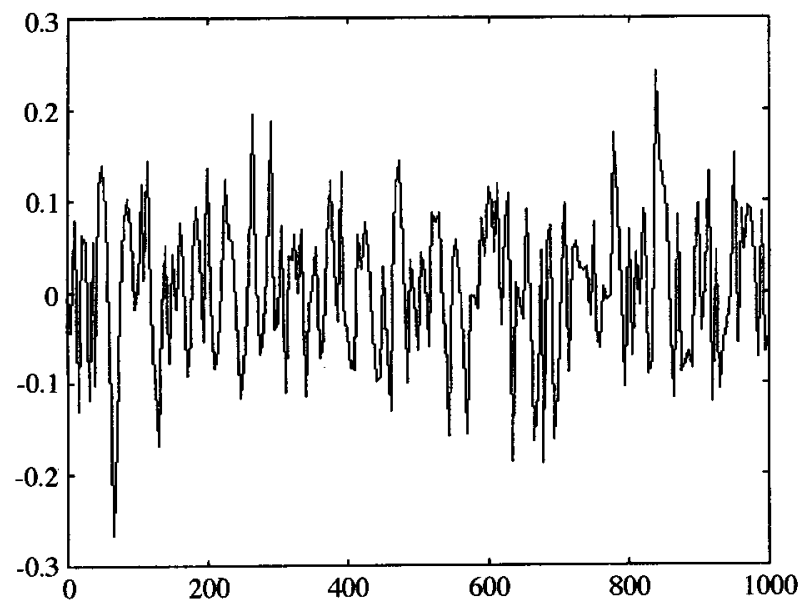

Fig. 6. Output response of heading angle $\psi(t)$ controlled by dynamic controller (41) 
For demonstrating the usefulness of the present approach, we try to use other control technique to design controllers for achieving the same performance constraints $<1>-<3>$. Here, we will consider the minimum variance $L Q G$ technique. Now, we first define a minimum variance cost function for the system model:

$J_{L Q G}=\lim _{t \rightarrow \infty} E_{\hat{t}}^{1} \int_{0}^{t}\left[\hat{x}^{T}(t) \hat{Q} \hat{x}(t)+u^{T}(t) \hat{R} u(t)\right] d t$

where $\hat{x}=[r(t) \psi(t)]^{T}, \hat{Q}$ and $\hat{\boldsymbol{R}}$ are semi-positive definite weighting matrices for states and control inputs, respectively. Using the minimum variance LQG method $[17,28]$, one may obtain a static state feedback controller

$$
u(t)=-\hat{R}^{-1} B^{T} \hat{P} \hat{x}(t)=-K_{L Q G} \hat{x}(t),
$$

where $\hat{\boldsymbol{P}}$ is a symmetric positive definite matrix which satisfy the following Riccati equation:

$$
\hat{\boldsymbol{P} A}+\boldsymbol{A}^{\boldsymbol{T}} \hat{\boldsymbol{P}}-\hat{\boldsymbol{P}} \boldsymbol{B} \hat{\boldsymbol{R}}^{-1} \boldsymbol{B}^{\boldsymbol{T}} \hat{\boldsymbol{P}}+\hat{\boldsymbol{Q}}=0 .
$$

The cost function considered here is a minimum variance performance index. For achieving the variance constraints $<1>$, we try to design some minimum variance $L Q G$ controllers subject to different weighting matrices. These results will stated as follows.

\section{Case I:}

If we choose $\hat{\boldsymbol{R}}=1$ and $\hat{\boldsymbol{Q}}=\boldsymbol{I}_{2}$, where $\boldsymbol{I}_{2}=\left[\begin{array}{ll}1 & 0 \\ 0 & 1\end{array}\right]$, then the static state feedback gain $K_{L Q G}=\left[\begin{array}{ll}K_{1} & K_{2}\end{array}\right]=$ [ $-86.8547-1]$ will be obtained by solving Riccati Eq. (44). From the simulations (Figs. 7 and 8), one may obtain that $E\left[r^{2}(\mathrm{t})\right]=3.3792, E\left[\psi^{2}(\mathrm{t})\right]=3507.6$, $\|H(s)\|_{\infty}=1601.0273$ and the closed-loop poles are $-0.0193+j 0.0158,-0.0193-j 0.0158$. It is easy to find that the performance requirements $<1>-<3>$ are all not satisfied in this case.

\section{Case II:}

In Case I, the variances of states are too large. Hence, we must increase the values of weighting matrix $\hat{\boldsymbol{Q}}$ to reduce the state variances. If we choose $\hat{\boldsymbol{R}}=1$ and $\hat{\boldsymbol{Q}}=2 \times 10^{6} I_{2}$, then we have $K_{L Q G}=$ [-2579.6249-1414.2136]. Using this controller to drive the system model, one may obtain that $E\left[r^{2}(\mathrm{t})\right]=0.0116, E\left[\psi^{2}(\mathrm{t})\right]=0.0753$ (Figs. 9 and 10 ), $\mid \boldsymbol{H}(s) \|_{\infty}=2.3028$ and the closed-loop poles are $-0.7983+j 0.4966,-0.7983-j 0.4966$. The variance constraints are achieved, however, the $\boldsymbol{H}_{\infty}$ norm and pole location constraints are all not satisfied.

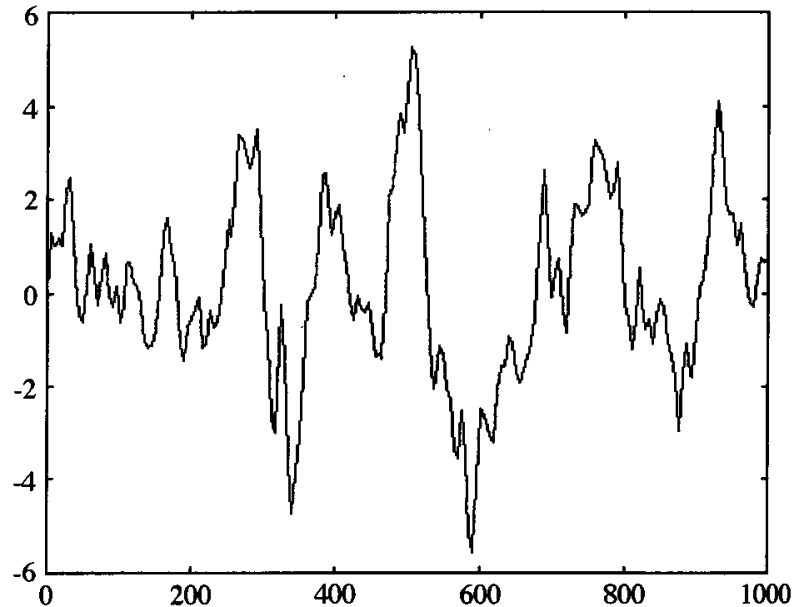

Fig. 7. Output response of yaw rate $r(t)$ controlled by LQG controller (Case I).

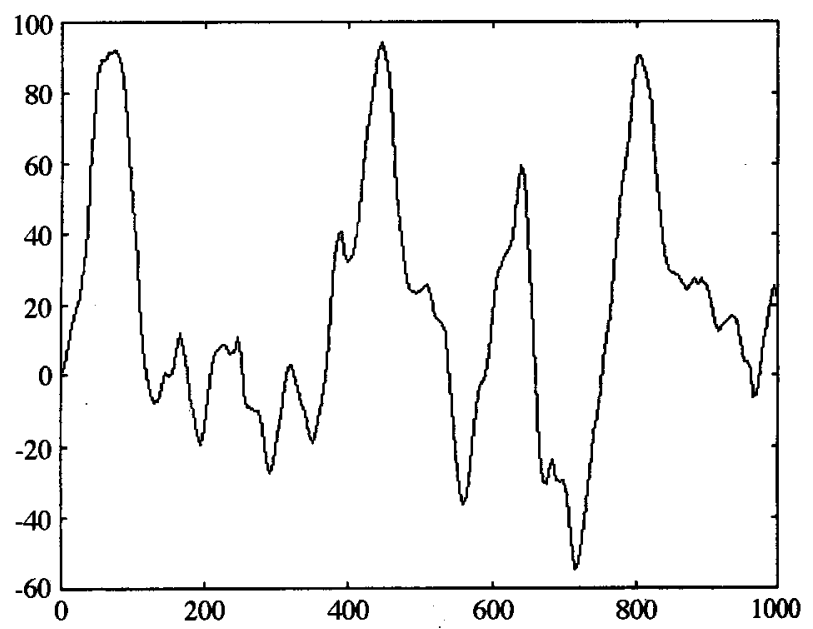

Fig. 8. Output response of heading angle $\psi(t)$ controlled by LQG controller (Case I).

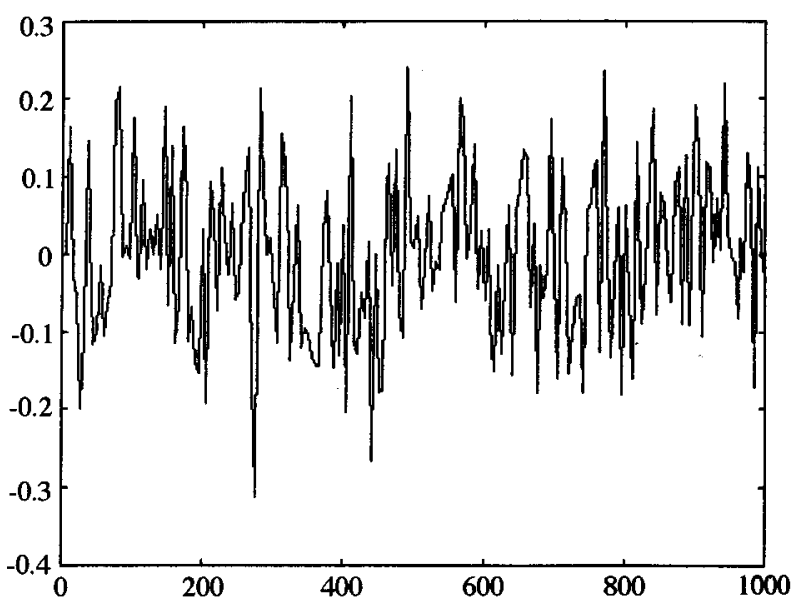

Fig. 9. Output response of yaw rate $r(t)$ controlled by LQG controller (Case II). 


\section{Case III:}

For comparing the LQG controller with the dynamic controller, we increase the values of weighting matrix $\hat{\boldsymbol{Q}}$ once again in order to obtain similar state variance values which have been got by using dynamic controller Eq. (41). If we choose $\hat{\boldsymbol{R}}=1$ and $\hat{Q}=2 \times 10^{8} I_{2}$, then we have $K_{L Q G}=[-15685.634$ $-1414.1356]$. From the simulated response (Figs. 11 and 12), we obtain that $E\left[r^{2}(t)\right]=0.007492, E\left[\psi^{2}(t)\right]$ $=0.009395, \mid H(s) \|_{\infty}=1.4942$ and the closed-loop poles are -8.7814 and -1.0065 . The variance constraints are approach to that obtained by using dynamic controller Eq. (41). However, the $\boldsymbol{H}_{\infty}$ norm and pole location constraints are still not achieved.

To summarize the above situations, we provide a summary table as Table 1 .

From the above table, we can find that the results of Case III is the best one between the above three LQG control simulations. Hence, let us compare the results of LQG control simulations (Case III) with that of dynamic control simulations. The state variances of these two situations are similar and are both satisfied constraints $<1>$. Though, a closed-loop pole of LQG control (Case III) is satisfied the constraints $\langle 3\rangle$, the other one is not. Moreover, the $\boldsymbol{H}_{\infty}$ norm constraints $<2>$ are still not achieved in Case III. Note that the values of weighting matrix $\hat{\boldsymbol{Q}}$ is very large in Case III. Furthermore, the control gain values of LQG controller (Case III) are larger than that of dynamic control gain Eq. (40). According to the same state variance values, dynamic controllers propose a small control gain as well as simultaneous satisfactions for individual state variance constr aints, $\boldsymbol{H}_{\infty}$ norm constraints and pole placement constraints. Hence, we may conclude that the dynamic controller design method presented in this paper provide a better system performance than that controlled by the minimum variance LQG technique.

\section{CONCLUSIONS}

The multiple constrained dynamic controller design for ship steering yaw-control problem has been considered in this paper. For this problem, we have introduced a methodology to derive the necessary and sufficient conditions for the existence of the dynamic controllers. Moreover, the controller solutions are also solved by using the upper bound covariance assignment technique. It has been shown, based on a numerical simulation of a simple ship steering model, that the present approach provides a set of dynamic controllers such that the individual state variance constraints, $\boldsymbol{H}_{\infty}$ norm constraints and

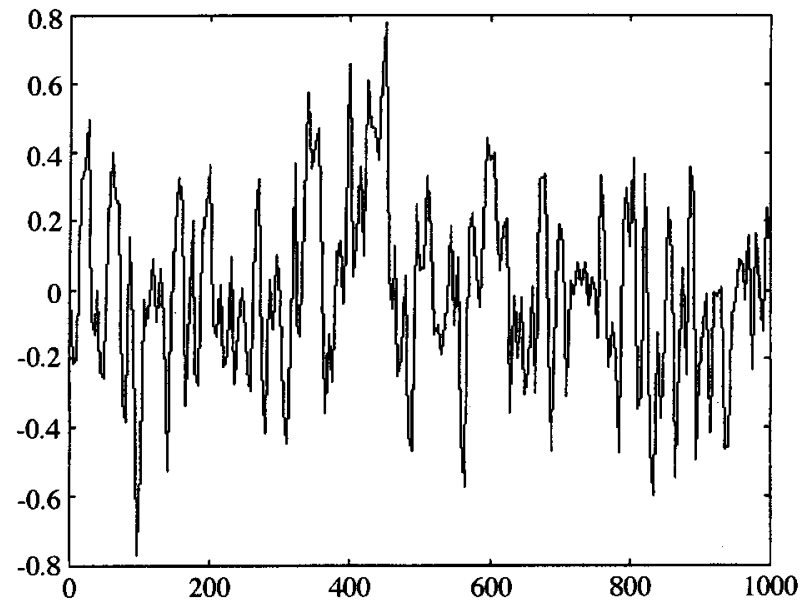

Fig. 10. Output response of heading angle $\psi(t)$ controlled by LQG controller (Case II).

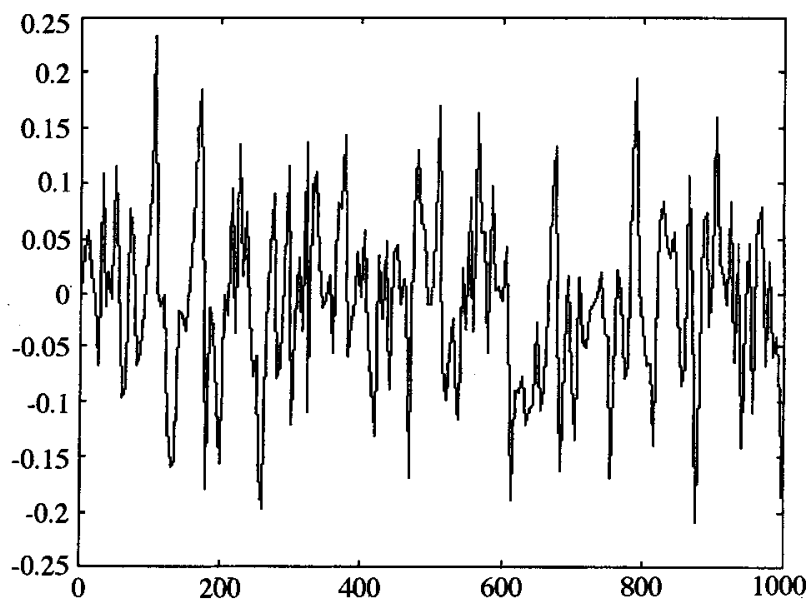

Fig. 11. Output response of yaw rate $r(t)$ controlled by LQG controller (Case III).

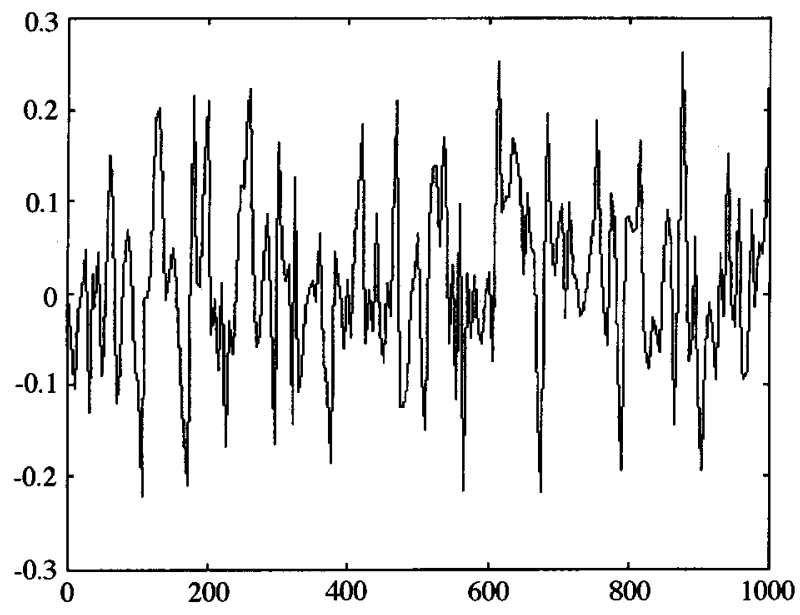

Fig. 12. Output response of heading angle $\psi(t)$ controlled by LQG controller (Case III). 
Table 1. Simulation comparison between different controllers

\begin{tabular}{|c|c|c|c|c|}
\hline & $\begin{array}{l}\text { LQG Controller (Case I) } \\
\qquad \hat{Q}=I_{2}\end{array}$ & $\begin{array}{l}\text { LQG Controller (Case II) } \\
\hat{Q}=2 \times 10^{6} I_{2}\end{array}$ & $\begin{array}{l}\text { LQG Controller (Case III) } \\
\hat{Q}=2 \times 10^{8} I_{2}\end{array}$ & $\begin{array}{l}\text { Dynamic Controller } \\
\text { (present approach) }\end{array}$ \\
\hline & $K_{L Q G}=\left[\begin{array}{ll}K_{1} & K_{2}\end{array}\right]$ & $K_{L Q G}=\left[\begin{array}{ll}K_{1} & K_{2}\end{array}\right]$ & $K_{L Q G}=\left[\begin{array}{ll}K_{1} & K_{2}\end{array}\right]$ & \\
\hline \multirow[t]{2}{*}{ Control Gain } & $K_{1}=-86.8547$ & $\boldsymbol{K}_{\mathrm{I}}=-2579.6249$ & $K_{1}=-15685.6341$ & Equation (41) \\
\hline & $K_{2}=-1$ & $K_{2}=-1414.2136$ & $K_{2}=-14142.1356$ & \\
\hline$E\left[r^{2}(t)\right]$ & 3.3792 & 0.0116 & 0.0074592 & 0.00797582 \\
\hline$E\left[\psi^{2}(t)\right]$ & 3507.6 & 0.0753 & 0.0093495 & 0.008637664 \\
\hline$\|\boldsymbol{H}(\boldsymbol{s})\|_{\infty}$ & 1601.0273 & 2.3028 & 1.4942 & 0.2075 \\
\hline \multirow[t]{3}{*}{ closed-loop poles } & $-0.0193+\mathrm{j} 0.0158$ & $-0.7983+\mathrm{j} 0.4966$ & -8.7814 & -2.0046 \\
\hline & $-0.0193-\mathrm{j} 0.0158$ & $-0.7983-j 0.4966$ & -1.0065 & $-2.3528+\mathrm{j} 0.5743$ \\
\hline & & & & $-2.3528-\mathrm{j} 0.5743$ \\
\hline Meet Constraint $<1>$ & No & Yes & Yes & Yes \\
\hline Meet Constraint $<2>$. & No & No & No & Yes \\
\hline Meet Constraint $<3>$ & No & No & No & Yes \\
\hline
\end{tabular}

$D(\alpha, \rho)$-stability constraints are satisfied, simultaneously. The system considered in this paper is a nominal one. Hence, it will be worthy to extend the present approach to the perturbed ship steering systems in the future.

\section{ACKNOWLEDGEMENTS}

The authors wish to express sincere thanks to the anonymous referees for their constructive comments and suggestions which have made substantial improvements on this paper. This work was supported by the National Science Council of the Republic of China under Contract NSC86-2213-E-019005 .

\section{REFERENCES}

1. Aneronger, J.V., "Adaptive Steering of Ships - A Model Reference Approach," Automatica, Vol. 20, pp. 3-14 (1984).

2. Kallstrom, C.G., "Identification and Adaptive Control Applied to Ship Steering," Department of Automatic Control, Lunb Institute of Technology, Lunb, Sweden, CODEN: LUTFD2/(TFRT-1018)/1-192 (1979).

3. Astrom, K.J., "Why Use Adaptive Techniques for Steering Large Tankers?' Int. J. Control, Vol. 32, No. 4, pp. 689-708 (1980).

4. Fung, P.T.K. and Grimble, M.J., "Dynamic Ship Positioning Using a Self-Tuning Kalman Filter," IEEE Trans. Auto. Control, Vol. AC-28, No. 3 pp. (1983).

5. Ohtsu, K., Horigome, M. and Kitagawa, G., “A New Ship's Auto Pilot Design Through a Stochastic Model," Automatica, Vol. 15, pp. 255-268 (1979).

6. Reid, R.E., Tugcu, A.K. and Mears, B.C., "The Use of Wave Filter Design in Kalman Filter State Estimation of the Automatic Steering Problem of a Tanker in a Seaway," IEEE Trans. Auto. Control, Vol. AC-29, No. 7, pp. (1984).

7. Reid, R.E., et al, "Comparison of Automatic Steering Performance of a VLCC in a Seaway Resulting from Application of LQG and Classical Control System Design Techniques," Proc. 6th Ship Control System Symposium, Vol. 4 (1981).

8. Amerongen, J. V. and Lemke, H. R. N., "Rudder Roll Stabilization", in Ship Operation Automation IV, North-Holland Publishing Company (1983).

9. Kallstrom, C.G., "Control of Yaw and Roll by a Rudder/Fin-Stabilization System," Proc. 6th Ship Control System Symposium, Vol. 2 (1981).

10. Zuidweg, J.K., "Optimal and Suboptimal Feedforward in Automatic Trackkeeping Systems," Proc. 6th Ship Control System Symposium, Vol. 2, (1981).

11. Francis, B.A., A Course in $\boldsymbol{H}_{\infty}$ Control Theory, Vol. 88 in Lecture Notes in Control and Information Sciences, Spring-Verlag, New York (1987).

12. Doyle, J., Glover, K., Khargonekar, P.P. and Francis, B.A., "State-Space Solutions to Standard $\boldsymbol{H}_{2}$ and $\boldsymbol{H}_{\infty}$ Control Problems," IEEE Trans. Auto. Control, Vol. 34, pp. 831-847 (1989).

13. Chu, C.C., Doyle, J.C. and Lee, E.B., "The General Distance in $\boldsymbol{H}_{\infty}$-Optimal Control Theory," Int. J. Control, Vol. 44, pp. 565-596 (1986).

14. Yaesh, I. and Shaked, U., "Minimum $\boldsymbol{H}_{\infty}-$ Norm Regulation of Linear Discrete-Time Systems and Its Relation to Linear Quadratic Discrete Games," IEEE Trans. Auto. Control, Vol. 35, No. 9, pp. 1061-1064 (1990).

15. Chang, W.J. and Chung, H.Y., "A Study of $\boldsymbol{H}_{\infty}$ Norm and Variance Constrained Design Using Dynamic Output Feedback for Linear Discrete Systems," Int. J. Control, Vol. 57, No. 2, pp. 473-483 (1993).

16. Chang, W.J. and Chung, H.Y., "Upper Bound Covariance Control with $\boldsymbol{H}_{\infty}$ Performance Bound for Linear 
Continuous Systems with Structure Uncertainty,"J. of the Chinese Institute of Electrical Engineering, Vol.2, No. 2, pp. 119-129 (1995).

17. Haddad, W.M. and Bernstein, D.S., "Controller Design with Regional Pole Constraints," IEEE Trans. Auto. Control, Vol. 37, No. 1, pp. 54-69 (1992).

18. Furuta, K. and Kim, S.B., "Pole Assignment in a Specified Disk," IEEE Trans. Auto. Control, Vol. AC32, No. 5, pp. 423-427 (1987).

19. Kim, S.B. and Furuta, K., "Regulator Design with Poles in a Specified Region," Int. J. Control, Vol. 47, No. 1, pp. 143-160 (1988).

20. Zhang, H. and Shu, S.G., "Analytic Approach to Quadratic Control with Prescribed Relative Stability," Int. J. Control, Vol. 48, No. 5, pp. 1843-1850 (1988).

21. Chung, H.Y. and Chang, W. J., "Covariance Control with Variance Constraints for Continuous Perturbed Stochastic Systems," Systems \& Control Letters, Vol. 19, No. 5, pp. 413-417 (1992).

22. Chang, W.J. and Chung, H.Y., "A Covariance Controller Design Incorporating Optimal Estimation for Nonlinear Stochastic Systems," ASME, J. Dynamic Systems, Measurement and Control, Vol. 118, No. 2, pp. 346-349 (1996).

23. Chang, W.J. and Chung, H.Y., "Upper Bound Covariance Control of Discrete Perturbed Systems," Systems \& Control Letters, Vol. 19, No. 6, pp.493-498 (1992).

24. Willems, J.C., "Least Squares Stationary Optimal Control and the Algebraic Riccati Equation," IEEE Trans. Auto. Control, Vol. AC-16, pp.621-634(1971).

25. Horn, R.A. and Johnson, C.R., Matrix Analysis, Cambridge University Press (1985).
26. Xu, J.H. and Skelton, R.E., "An Improved Covariance Assignment Theory for Discrete Systems," IEEE Trans. Auto. Control, Vol. 37, No. 10, pp. 1588-1591 (1992).

27. Rao C.R. and Mitra, S.K., Generalized Inverse of Matrices and Its Applications, Wiley, New York (1971).

28. Anderson, B.D.O. and Moore, J.B., Optimal Control (Linear Quadratic Methods), Prentice-Hall Int., Inc. (1989).

\section{满足行為限制之船舶平摺操控系統 動態控制器設計}

$$
\text { 張 文 哲 }
$$

國立台灣海洋大學輪機工程技衍系

$$
\text { 摘 要 }
$$

本諭文探討满足多重行為限制之船舶平掍運 動操控系統的動態控制器設計問題, 本文中所論及 之多重行為限制包括個別狀態之方差限制、強健性 行為 $\left(H_{\infty}\right.$ 模) 限制及區域極點指定 (D-稳定) 限 制。存在满足以上三個行為限制之動態控制器的充 分及必要條件將首先在文中被推導得到, 當這些條 件满足時, 我們可以利用奇異值分解(SVD)技街及 廣義式逆求解理論來求得此動態控制器之解。最 後, 我們將用一個数值實例來說明本方法的有效性 及可應用性。 